\title{
Time Profile of the Neutrino Burst from the Supernova 1987A
}

\author{
Hideyuki Suzuki and Katsuhiko Sato \\ Department of Physics, Faculty of Science, University of Tokyo \\ Bunkyo-ku, Tokyo 113, Japan
}

SN1987A gave us the first opportunity to study the supernova core directly by providing us the neutrino signal from the core. The observational data of the neutrino flux detected by Kamiokande[1] and IMB[2] show surprisingly good agreements with the theoretical predictions as a. whole[3,4]. The fundamental concept of the collapse driven supernova explosion is confirmed for the first time. On the other hand, there are some puzzles. The most peculiar feature of the data is the 7 seconds gap of the Kamiokande data. The first 8 events of Kamiokande were detected in 2 seconds, following the 7 seconds gap and the last 3 events in 4 seconds. Of course just only 7 seconds gap is not unnatural if small neutrino flux come. But there were detected 3 events after the gap. These 3 events may not be produced by the weak flux. We can estimate the time integrated luminosity of $\bar{\nu}_{\mathrm{e}}$ corresponding to the last 3 events and get the large value such as $7 \cdot 10^{52} \mathrm{erg}$ [5]. Can we get out of this inconsistency, 3 events after the 7 seconds gap? If not, we may need to consider some nonstandard mechanism of the neutrino emission at the late time. In order to investigate the probability of the case in which there is a 7 seconds gap before 3 events, we have performed Monte Carlo simulations for the simple model of neutrino flux.

Simple Model of Neutrino Flux : Many authors study the data of Kamiokande and IMB and insist that their simple models of the neutrino flux can well explain the data. For example, Spergel et al. [6] investigate the cooling model of the protoneutron star and got the best fit parameters such as the radius, $R$, the initial neutrino temperature, $T_{i}$, and the decay time of the temperature, $\tau_{T}$. Note that most of them did not consider the contraction of the protoneutron star. It is considered that the protoneutron star born with the radius of several times $10 \mathrm{~km}$ contracts to the normal neutron star with the radius of about $10 \mathrm{~km}$ in a few seconds with emitting neutrinos and losing the thermal energy. Therefore we introduce one more parameter, the decay time of the radius, $\tau_{R}$. Consequently the number flux of $\bar{\nu}_{\mathrm{e}}, F(E, t)$ is given by

$$
\begin{array}{rlrl}
F(E, t) & =\frac{c}{4} \frac{4 \pi}{(h c)^{3}} \frac{E^{2}}{\exp [E / T(t)]+1} \frac{R(t)^{2}}{d^{2}} & & \left(t<t_{m}\right) \\
& =0 & & \left(t>t_{m}\right) \\
T(t) & =T_{i} \cdot \exp \left(-t / \tau_{T}\right) & \\
R(t) & =R_{f}+\left(R_{i}-R_{f}\right) \cdot \exp \left(-t / \tau_{R}\right) & &
\end{array}
$$

where $R_{i}, R_{f}$ are the initial and final radius respectively and the distance of SN1987A, $d$ is assumed to be $50 \mathrm{kpc}$.

Probability of 7 Seconds Gap before 3 Events : Using the above models of the neutrino flux, we have done Monte Carlo simulations for the Kamiokande detector. Then we investigate the 
Table I : Results of Monte Carlo Simulations.

\begin{tabular}{ccccccccc}
\hline $\begin{array}{c}T_{i} \\
(\mathrm{MeV})\end{array}$ & $\begin{array}{c}\tau_{T} \\
(\mathrm{sec})\end{array}$ & $\begin{array}{c}R_{i} \\
(\mathrm{~km})\end{array}$ & $\begin{array}{c}R_{f} \\
(\mathrm{~km})\end{array}$ & $\begin{array}{c}\tau_{R} \\
(\mathrm{sec})\end{array}$ & $\begin{array}{c}t_{m} \\
(\mathrm{sec})\end{array}$ & \multicolumn{1}{c}{$N$} & $\begin{array}{c}E_{\bar{\nu}} \\
\left(10^{52} \mathrm{erg}\right)\end{array}$ & Probability \\
\hline 4.2 & 18 & 27 & 27 & $\infty$ & 16 & 17.5 & 5.6 & $1 / 2813$ \\
4.2 & 18 & 20 & 10 & 10 & 16 & 7.5 & 2.3 & $1 / 2891$ \\
4.2 & 18 & 20 & 20 & $\infty$ & 13 & 9.5 & 3.0 & $0 / 7083$ \\
4.2 & 18 & 37 & 10 & 1 & 16 & 7.9 & 2.1 & $2 / 3505$ \\
4.2 & 18 & 40 & 20 & 1 & 16 & 15.5 & 4.5 & $1 / 5633$ \\
4.6 & 18 & 20 & 10 & 1 & 13 & 6.3 & 1.6 & $0 / 1611$ \\
4.6 & 18 & 20 & 10 & 10 & 16 & 12.4 & 3.2 & $2 / 8409$ \\
4.6 & 18 & 20 & 20 & $\infty$ & 13 & 15.7 & 4.3 & $1 / 5350$ \\
4.6 & 18 & 37 & 10 & 1 & 16 & 12.9 & 3.1 & $3 / 9862$ \\
5.0 & 18 & 20 & 10 & 1 & 16 & 9.8 & 2.3 & $6 / 6261$ \\
5.0 & 18 & 20 & 10 & 10 & 16 & 19.3 & 4.5 & $1 / 1413$ \\
5.0 & 18 & 37 & 10 & 1 & 16 & 19.9 & 4.3 & $0 / 1054$ \\
\hline
\end{tabular}

$N$ is the expected number of events in the Kamiokande detector and $E_{\bar{\nu}}$ is the total energy emitted as $\bar{\nu}_{\mathrm{e}}$.

probability of the case in which there is a long gap longer than 7 seconds before the last 3 events. The results are summarized in Table I. Spergel et al.'s best fit flux is also included. In 2813 trials with their model, there is only one trial in which 3 events occurred after 7 seconds gap. In all other cases the probability is also very small, less than 0.1 percent. It seems that the probability is not very sensitive to the decay time of the radius. It is reasonable when we consider the dependence of the event rate to the temperature and the radius. The event rate is proportional to the neutrino number flux and the cross section of the reaction, $\bar{\nu}_{\mathrm{e}}+\mathrm{p} \longrightarrow \mathrm{e}^{+}+\mathrm{n}$, and the detection efficiency. The radius affects only the number flux and if the temperature remains constant the event rate is not reduced by the factor less than $\left(R_{f} / R_{i}\right)^{2}$. On the other hand the temperature strongly affects the event rate. The number flux, the cross section and the efficiency vary in the manner proportional to $T^{3}, T^{2}, T^{\alpha}$ respectively where $\alpha$ is some positive value. Consequently the event rate decays rapidly in the time scale of $\tau_{T}^{5+\alpha}$ and it becomes very difficult for the flux to produce more than 3 events after the long gap. Of course more systematic analysis is required, but it is cleared by this analysis that the rapidly decreasing $\bar{\nu}_{\mathrm{e}}$ flux is inconsistent with the data of Kamiokande. If the protoneutron star actually cools down rapidly, the Kamiokande data indicate some unknown mechanism of neutrino emission 10 seconds after the collapse. We must be allowed to consider some nonstandard mechanism of the neutrino emission.

Note added : After this colloquium, we analyze the the probability of the long gap systematically. We find that the almost constant neutrino flux whose decay time of the event rate is longer than 10 seconds can caused the 7 seconds gap before 3 events with the probability of $1 \sim 2$ percent. But it seems not very natural to consider the constant neutrino flux.

\section{References}

[1] K. Hirata et al., Phys. Rev. Lett. 58 (1987) 1490.

[2] R. Bionta et al., Phys. Rev. Lett. 58 (1987) 1494.

[3] K. Sato and H. Suzuki, Phys. Rev. Lett. 58 (1987) 2722.

[4] K. Sato and H. Suzuki, Phys. Lett. in press.

[5] H. Suzuki and K. Sato, Publ. Astron. Soc. Japan 39 (1987) 521.

[6] D. N. Spergel et al. preprint (1987). 